This study examines a concurrent profiling of circulating and extravasated polymorphonuclear leukocytes (PMNs) in a rat model of experimental sepsis. Fecal peritonitis was induced in Wistar male rats by intraperitoneal instillation of a fecal suspension in saline $(1: 1 \mathrm{w} / \mathrm{v})$. Blood and peritoneal fluid were collected $8 \mathrm{~h}$ following fecal inoculation for the evaluation of inflammatory response of PMNs using zymosan-induced luminol-dependent chemiluminescence. Fifty microliters of pre-diluted blood or peritoneal fluid samples were mixed with $150 \mu 1$ of reaction mixture $\left(4 \times 10^{-4} \mathrm{M}\right.$ luminol $+50 \mu \mathrm{g}$ opsonized zymosan $+0.1 \%$ gelatin in Hank's balanced salt solution) and the chemiluminescence signal was measured in a luminometer at $37^{\circ} \mathrm{C}$. Fecal peritonitis caused a significant leukocytopenia $(3540 \pm 297$ $\mathrm{mm}^{-3}$ versus control value of $7525 \pm 711 \mathrm{~mm}^{-3}$, $p<0.001$ ) accompanied by massive infiltration of PMNs in the peritoneal cavity $(34700 \pm 4006$ versus $\left.7325 \pm 425 \mathrm{~mm}^{-3}, p<0.001\right)$. The phagocytic activity of circulating blood PMNs was down-regulated whereas a significant up-regulation was observed in the activity of PMNs from peritoneal fluid. In conclusion, this study clearly demonstrates sepsis-induced alterations in both blood and peritoneal fluid PMNs and their quantitative assessment may be helpful in disease evaluation and designing effective therapies.

Key words: Septicemia, Peritonitis, Leukocytes, Phagocytosis, Chemiluminescence, Rat

\section{Zymosan-induced luminol- dependent chemiluminescence response of circulating and extravasated leukocytes in experimental sepsis}

\author{
Haseeb Ahmad Khan ${ }^{\text {CA }}$
}

Research Center, Riyadh Al Kharj Hospital Program, P.O. Box 7897 (T-835), Riyadh 11159, Saudi Arabia

\author{
${ }^{\mathrm{CA}}$ Corresponding author \\ Tel: +9661 4777714, ext. 3809 \\ E-mail: khan_haseeb@yahoo.com
}

\section{Introduction}

Peritonitis is a serious infection that often leads to multiple organ failure, septicemia and mortality. The cascade of events initiating from infection to septic shock and organ failure is poorly understood. Phagocytosis is the first line of host defense against foreign antigens including bacteria. Polymorphonuclear leukocytes (PMNs) such as neutrophils and macrophages are responsible for inactivating and clearing bacteria and microbial-related noxious products using the process of phagocytosis. The recruitment and activation of PMNs at the site of infection are essential for effective host defense. ${ }^{1}$ However, prolonged activation of PMNs results in the excessive generation of pro-inflammatory cytokines and reactive oxygen species (ROS) that can lead to progressive damage of host tissue. ${ }^{2}$ Activated PMNs have also been implicated in oxidant stress and altered biochemistry of red blood cells in sepsis. ${ }^{3}$

Recently, Holzer et al. ${ }^{4}$ suggested that immunomodulation to down-regulate circulating PMNs and to up-regulate emigrated PMNs might offer a valuable protection against complicated peritonitis. Thus, a concurrent evaluation of the phagocytic response of PMNs in blood and peritoneal fluid could help in grading septicemia and planning more effective pharmacotherapies. In this investigation, an attempt has been made to evaluate phagocytic response of PMNs in the blood and peritoneal fluid of rats subjected to fecal peritonitis.

\section{Materials and methods}

\section{Animals}

Wistar male rats $(230 \pm 10 \mathrm{~g})$ were divided into two groups of five animals each. The rats were housed in a temperature-controlled room maintained in $12 \mathrm{~h}$ light/dark cycles. The standard laboratory food and water were freely available ad libitum except for an overnight fasting before inducing experimental sepsis in rats. The study protocol was supported by the Research and Ethics Committee of Riyadh Al-Kharj Hospital Program, Saudi Arabia.

\section{Experimental sepsis model}

A small incision was made in the abdomen of etheranesthetized rats for intraperitoneal instillation of fecal suspension at a dosage of $1 \mathrm{ml} / \mathrm{kg}$ body weight of animals. The fecal suspension was prepared by 
dissolving fresh feces ( $1: 1 \mathrm{w} / \mathrm{v}$ in normal saline) obtained surgically from the caecum of non-fasted healthy rats, and was used within $2 \mathrm{~h}$. Control animals received pre-autoclaved $\left(135^{\circ} \mathrm{C}\right.$ for $\left.1 \mathrm{~h}\right)$ fecal suspension. The wound was closed and the animals returned to their home cages. The surgery was performed aseptically. This procedure results in a fecal peritonitis septicemia. ${ }^{5}$

\section{Blood and peritoneal fluid samples}

The specimens of blood and peritoneal fluid were collected at $8 \mathrm{~h}$ following fecal inoculation. The samples were diluted either 500-fold (blood) or 100-fold (peritoneal fluid) in Hank's balanced salt solution (HBSS) containing $0.1 \%$ gelatin, and were kept on ice until promptly analyzed.

\section{Measurement of phagocytosis}

A sensitive procedure based on zymosan-induced luminol-enhanced chemiluminescence (CL) was used to measure the phagocytic response of leukocytes. ${ }^{6}$ Fifty microliters of pre-diluted blood or peritoneal fluid samples were mixed with $150 \mu$ of reaction mixture $\left(4 \times 10^{-4} \mathrm{M}\right.$ luminol $+50 \mu \mathrm{g}$ opsonized zymosan $+0.1 \%$ gelatin in HBSS) in the well of an opaque cliniplate (Labsystems, Helsinki, Finland). The CL signal produced by phagocytosing leukocytes was measured in a luminometer (Model LuminoscanRT; Labsystems) at $37^{\circ} \mathrm{C}$. Thirty cycles of measurements using a $5 \mathrm{sec}$ counting time and a $70 \mathrm{sec}$ interval time were performed for each sample. Leukocytes were counted in all the samples using a hemocytometer.

\section{Statistics}

The statistical significance between the control and sepsis groups was analyzed by independent sample $t$-test. $p<0.05$ was considered statistically significant.

\section{Results}

The leukocyte count was significantly decreased in the blood and increased in the peritoneal fluid of animals that underwent peritoneal fecal instillation

Table 1. Effect of experimental peritonitis on leukocytes count in blood and peritoneal fluid

\begin{tabular}{|c|c|c|}
\hline \multirow{2}{*}{$\begin{array}{l}\text { Experimental group } \\
(n=5)\end{array}$} & \multicolumn{2}{|c|}{ Leukocyte count $\left(\mathrm{mm}^{-3}\right)$} \\
\hline & Blood & Peritoneal fluid \\
\hline $\begin{array}{l}\text { Control } \\
\text { Peritonitis }\end{array}$ & $\begin{array}{l}7525 \pm 711 \\
3540 \pm 297^{*}\end{array}$ & $\begin{array}{c}7325 \pm 425 \\
34,700 \pm 4006 *\end{array}$ \\
\hline
\end{tabular}

* $p<0.001$ versus the respective control groups ( $t$-test).
(Table 1). The peak CL response during the entire counting time was not significantly different between the control and peritonitis groups (Table 2), whereas a significant decrement in the integrated CL signals was observed in the peritonitis group as compared with the CL response from the same volume $(100 \mathrm{nl})$ of control blood. The normalization of CL to 1000 leukocytes also showed reduced phagocytic response in the peritonitis group but without reaching a significance level (Table 2). In contrast to blood analysis, all the CL measurements (peak value, integral value, normalized value) were significantly higher in the peritoneal fluid of rats with septicemia as compared with respective control values (Table 2 ).

\section{Discussion}

A significant reduction in blood leukocyte count following experimental sepsis is in agreement with earlier studies. ${ }^{3,7}$ Transient leukocytopenia has also been reported in patients with sepsis who developed respiratory failure, suggesting that the disappearance of PMNs from the blood stream and entrapment of cells in lung capillaries is an early event of acute respiratory distress syndrome..$^{8}$ On the other hand, fecal peritonitis caused a significant increase in the leukocyte count within the peritoneal fluid, indicating massive infiltration of PMNs towards the bacterial load. PMN migration into the peritoneal cavity in response to fecal peritonitis is an important mechanism of host defense against bacterial invasion as the exudative PMNs localize and contain infection by phagotizing and killing bacteria.?

The PMN migration to the peritoneal cavity showed a highly significant up-regulation of their phagocytic activity as opposed to a concurrent downregulation of phagocytic activity of blood PMNs (Table 2). These findings are supported by an earlier study showing the ability of ascitic PMNs to produce significantly high levels of ROS, including superoxide, hydrogen peroxide and myeloperoxidase, as compared with blood PMNs following induced peritonitis in rats. ${ }^{10}$ As the ROS have a tendency of producing CL in the presence of luminol, a high CL signal from migrated PMNs during sepsis indicates excessive generation of ROS that might trigger pathways for host damage. ${ }^{2,10}$

Activated PMNs are considered the primary mediators of remote organ injury in peritonitis., 11 The severe systemic inflammation caused by sepsis may also lead to multiple organ failure, a condition that is often fatal to the host. ${ }^{12}$ The natural resolution of acute inflammation involves bulk clearance of extravasated inflammatory cells in an ordered manner. However, an imbalance between the clearance and infiltration of PMNs and/or disruption of equilibrium 
Table 2. Phagocytic activity of leukocytes in blood and peritoneal fluid, measured by zymosan-induced luminol-dependent chemiluminescence response

\begin{tabular}{lccc}
\hline $\begin{array}{l}\text { Experimental group } \\
(n=5)\end{array}$ & $\begin{array}{c}\text { Peak RLU } \\
\left(\times 10^{-3}\right)\end{array}$ & $\begin{array}{c}\text { Integral RLU } \\
(30 \mathrm{~min})\end{array}$ & $\begin{array}{c}\text { RLU/1000 } \\
\text { leukocytes }\end{array}$ \\
\hline $\begin{array}{l}\text { Blood } \\
\quad \text { Control }\end{array}$ & $4.00 \pm 0.91$ & $4.576 \pm 1.61$ & $6.160 \pm 2.26$ \\
$\quad$ Peritonitis & $3.00 \pm 0.54$ & $0.895 \pm 0.42^{*}$ & $2.958 \pm 1.52$ \\
Peritoneal fluid & & & $5.21 \pm 2.16$ \\
$\quad$ Control & $12.50 \pm 4.80$ & $18.25 \pm 7.60$ & $22.56 \pm 5.93^{*}$ \\
\hline
\end{tabular}

RLU, relative light unit, an arbitrary unit of chemiluminescence measurement.

${ }^{*} p<0.05$ and ${ }^{* *} p<0.01$ versus the respective control group ( $t$-test).

between bacterial load and extent of phagocytosing PMNs could be deleterious to host cells. Therefore the role of PMNs should be evaluated in the light of both host defense as well as host injury, while approaches to control PMN infiltration to the infected site and regulation of their activity have improved the animals' survival and protected them against remote organ injury following experimental peritonitis. ${ }^{13-15}$

In conclusion, this study clearly showed differential changes in the blood and ascitic PMNs following fecal peritonitis. However, the real clinical application of these parameters in defining septicemia is yet to be established.

\section{References}

1. Mercer-Jones MA, Heinzelmann M, Peyton JC, Wickel D, Cook M, Cheadle WG. Inhibition of neutrophil migration at the site of infection increases remote organ neutrophil equestration and injury. Shock 1997; 8: $193-199$.

2. Goya T, Morisaki T, Torisu M. Immunologic assessment of host defense impairment in patients with septic multiple organ failure: relationship between compliment activation and changes in neutrophil function. Surgery 1994; 115: 145-155.

3. Baskurt OK, Temiz A, Meiselman HJ. Red blood cell aggregation in experimental sepsis. J Lab Clin Med 1997; 130: 183-190.

4. Holzer K, Richter A, Konietzny P, Schubel F, Wilhelm K, Henrich D. Functions of circulating and intra-abdominal polymorphonuclear leukocytes during human secondary peritonitis. Zentralbl Chir 2003 128: $291-297$.

5. Noronha-Blob N, Lowe VC, Otterbein L, Steranka L, Burch RM. NPC 15669 reduces mortality associated with sepsis in rats. J Pharmacol Exp Ther 1993; 267: 664-669.
6. Lilius E, Waris M. A very sensitive and rapid chemiluminescence method for the measurement of phagocytosis. Analytical Applications of Bioluminescence and Chemiluminescence. London: Academic Press, 1984: 401- 404

7. Jacobi CA, Ordemann J, Halle E, Volk HD, Muller JM. Impact of laparoscopy with carbon dioxide versus helium on local and systemic inflammation in an animal model of peritonitis. $J$ Laparoendosc Surg Tech A 1999; 9: 305-312

8. Thommasen HV, Russel JA, Boyko WJ, Hogg JC. Transient leukopenia associated with adult respiratory distress syndrome. Lancet 1984; 2 : $809-812$

9. Frode TS, Ferreira SI, Medeiros YS. Analysis of local and systemic inflammatory responses induced by polymicrobial peritonitis in mice. Mediat Inflamm 2001; 10: 237-243.

10. Shen SC, Nakao A, Kishimoto W, Harada A, Nonami T, Nakano M, Takagi $H$. The ability of polymorphonuclear leukocytes to produce active oxygen in a model of peritonitis in rats. Surg Today 1993; 23: 603-608.

11. Wickel D, Cheadle W, Mercer Jones M, Garrison R. Poor outcome from peritonitis is caused by disease acuity and organ failure, not recurrent peritoneal infection. Ann Surg 1997; 225: 744-753.

12. Bone RC. Immunologic dissonance: a continuing evolution in our understanding of the systemic inflammatory response syndrome (SIRS) and the multiple organ dysfunction syndrome. Ann Intern Med 1996; 125: $680-687$.

13. Gardinali $\mathrm{M}$, Borrelli $\mathrm{E}$, Chiara $\mathrm{O}$, et al. Inhibition of CD11-CD18 complex prevents acute lung injury and reduces mortality after peritonitis in rabbits. Am J Respir Crit Care Med 2000; 161: 1022-1029. 14. Tariq M, Al Moutaery A, Arshaduddin M, Khan HA, Price Evans D, Jacobs S. Fluconazole attenuates lung injury and mortality in rat peritonitis model. Intensive Care Med 2003; 29: 2043-2049.

15. Perdue PW, Kazarian KK, Nevola J, Law WR, Williams T. The use of local and systemic antibiotics in rat fecal peritonitis. J Surg Res 1994; 57: 360365.

Received 2 December 2003

Accepted 12 January 2004 


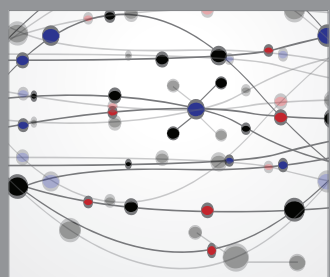

The Scientific World Journal
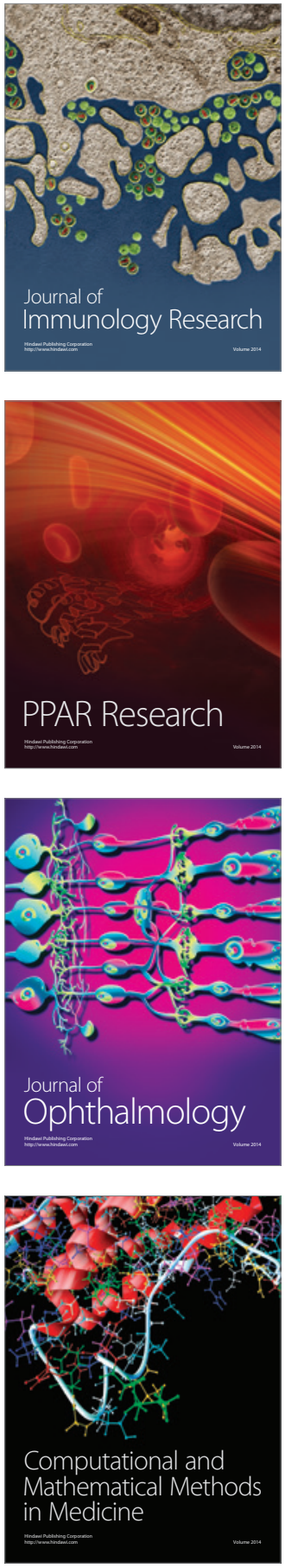

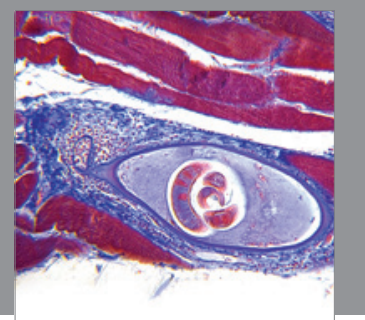

Gastroenterology

Research and Practice
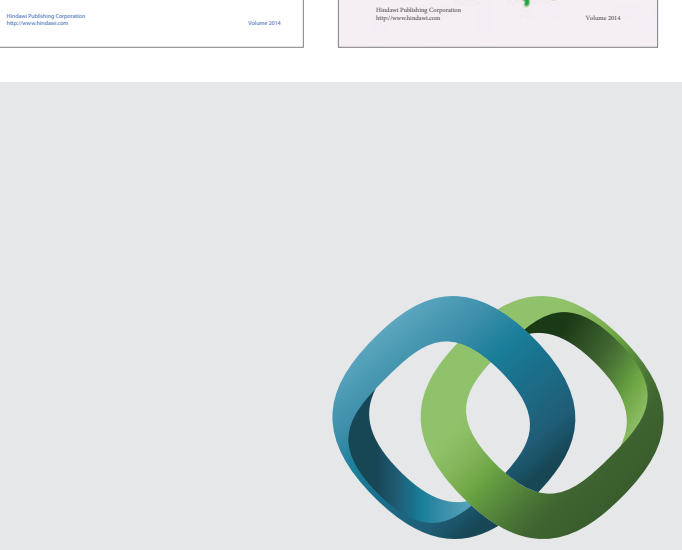

\section{Hindawi}

Submit your manuscripts at

http://www.hindawi.com
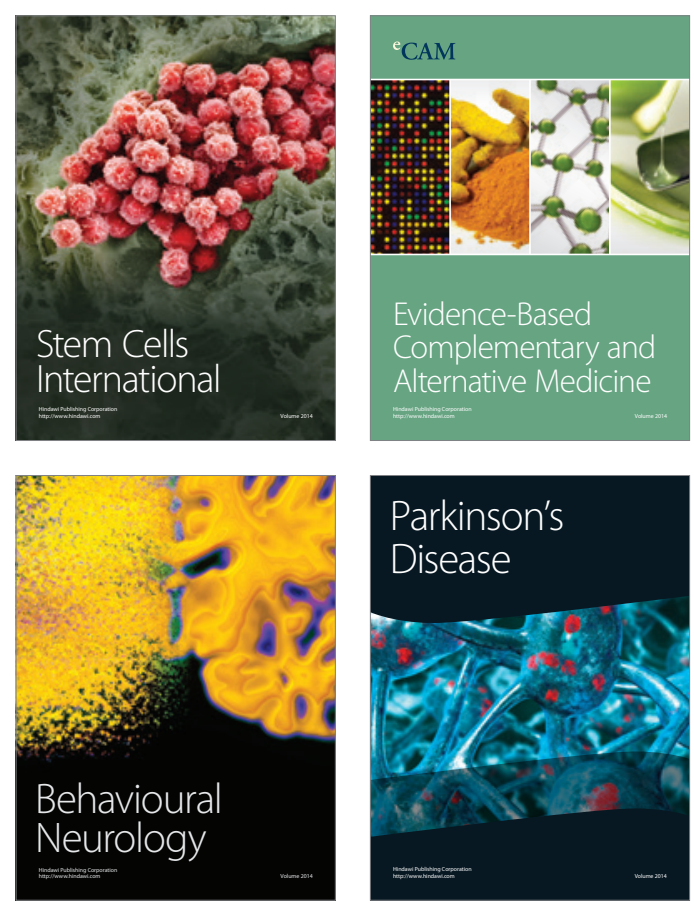

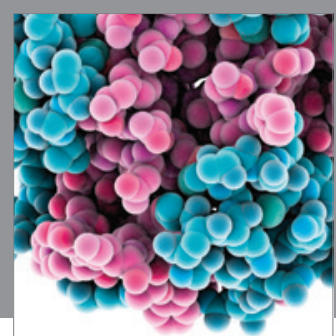

Journal of
Diabetes Research

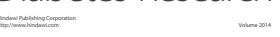

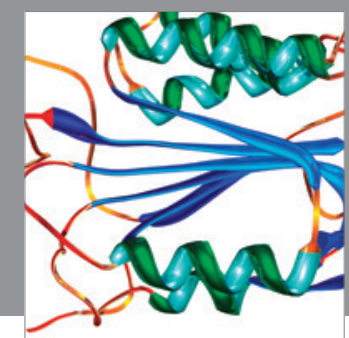

Disease Markers
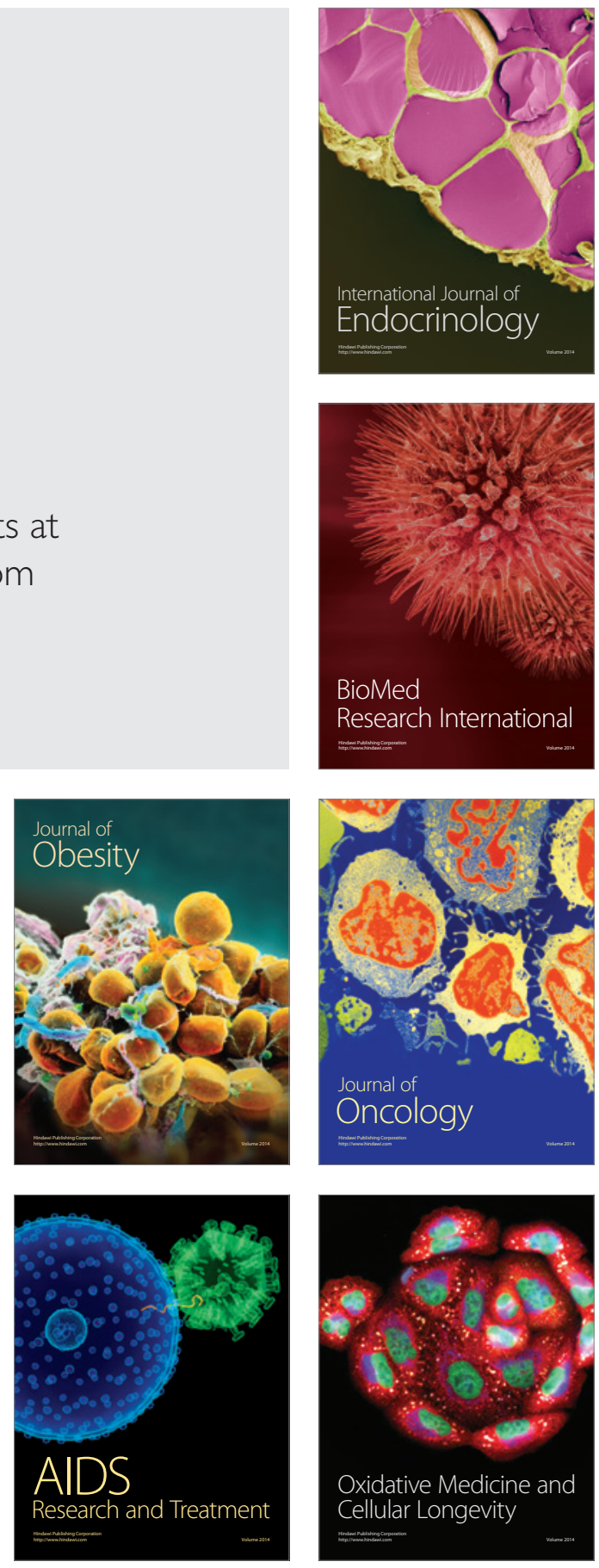